\title{
Early discharge after surgery for breast cancer was safe and well received by patients
}

\author{
Bonnema J, van Wersch AM, van Geel AN, et al. Medical and psychosocial effects of early discharge after surgery for breast cancer: \\ randomised trial. BMJ 1998 Apr 25;316:1267-71.
}

\section{Question}

Is early discharge after surgery for breast cancer associated with wound or drainage complications, changes in patient satisfaction, or psychosocial problems?

\section{Design}

Randomised controlled trial.

\section{Setting}

A general hospital and cancer clinic at a university hospital in Rotterdam, the Netherlands.

\section{Patients}

139 women with operable breast cancer (stage I or II) who were eligible for modified radical mastectomy or lumpectomy with axillary dissection. Exclusion criteria were radiotherapy or chemotherapy before surgery, or high risk of complications. Follow up was $90 \%$.

\section{Intervention}

Randomisation was done before admission. 69 women were allocated to early discharge (morning of day 4 after surgery). A discharge protocol, developed to guarantee continuity of care, included patient information, referral to a community health nurse, an emergency telephone number, and scheduled follow up visits. Drain removal was done in the outpatient clinic or at home. 70 women were allocated to long stay (usually 9-12 d) and were discharged after their drains were removed.

\section{Main outcome measures}

Complications included infection, necrosis, haematoma, wound dehiscence, or drain problems. Patient satisfaction was measured for length of stay, care received by community health nurse, and willingness to recommend short stay to others. Psychosocial function was measured using standard scales for uncertainty, state and trait anxiety, object anxiety, loneliness, depression, sleep disturbances, feeling of loss of control, self esteem, and cancer locus of control.

\begin{abstract}
Main results
The groups did not differ for duration or volume of drainage or complications except for a lower median number of percutaneous aspirations and more frequent leakage along the drain in the short stay group ( $p=0.04$ for both). $93 \%$ of patients in the short stay group felt satisfied with their care at home and $71 \%$ felt as secure at home as in the hospital. Over $90 \%$ of women in the short stay group and about $40 \%$ of women in the long stay group would recommend short stays to other patients. The groups did not differ for psychosocial problems, physical or psychological complaints, or coping strategies. Short stay patients were more likely to discuss their disease with their families.
\end{abstract}

\section{Conclusions}

Compared with usual discharge, early discharge after breast cancer surgery was assoicated with increased leakage along the drain and fewer percutaneous aspirations. Women who had early discharge were more likely to recommend a short stay to others and to discuss their disease with family members.

Source of funding: Ministry of Welfare, Health, and Sports, the Netherlands.

For correspondence: $\operatorname{Dr}$ J Bonnema, Department of Surgical Oncology, University Hospital Rotterdam/Daniel den Hoed Cancer Center, PO Box 5201, 3008 AE Rotterdam, the Netherlands. Fax +31104864645 .

\section{Commentary}

Bonnema et al clearly show that early discharge after surgery for breast cancer is not deleterious to patient health or recovery. Their findings are well supported by similar studies. ${ }^{1-3}$ The use of randomisation adds further credibility to the overall findings. Bonnema et al hypothesised that no difference would be shown between the 2 groups. Early discharge patients were, however, more likely to discuss their disease with their families. Judgment on the exact causal relation between early discharge and improved family communication must be reserved in the absence of direct assessment of this interaction.

One component of the discharge protocol was patient information provided by the breast cancer nurse. It would be interesting to know if family members were invited to take part in the educational preparation for discharge and whether they would have scored as high on satisfaction had they been assessed. The issue of inadequate sampling to detect small, but clinically significant, differences in wound complications could be addressed through a multicentre randomised trial.

Therapeutic and technological advances, coupled with economic constraints, have initiated a gradual move towards early discharge. This has many implications for the nursing profession, not just in oncology but across the healthcare continuum. This shift dictates the need for delivery of more comprehensive patient education about upcoming surgery, actual and potential complications after surgery, and projected recovery rate. Traditionally, patient education has been a nursing responsibility. This role is gaining importance in the context of the diminishing time frame in which nurses now practice. Nurses have a responsibility to coordinate patient care to achieve optimal clinical outcomes through the provision of continuous, high quality care that is both effective and individualised. It is therefore reassuring to find that early discharge of patients with breast cancer is not deleterious to patient recovery and may even result in a more positive experience.

Sharon Lowry, RGN, BSc Clinical Trials Co-ordinator Surgical Oncology Unit Beaumont Hospital Dublin, Ireland

\footnotetext{
1 Holcombe C, West N, Mansel RE, et al. Eur J Surg Oncol 1995;21:604-6.

2 Boman L, Bjorvell H, Cedermark B, et al. Eur J Surg 1993;159:67-73.

3 Clarke JA, Kent RB 3rd. Am Surg 1992;58:239_
} 